Diabetologe 2009 · 5:602-604

DOI 10.1007/s11428-009-0505-y

(c) Springer-Verlag 2009

\author{
F. Erbguth \\ Klinik für Neurologie, Klinikum Nürnberg (Süd), Nürnberg
}

\title{
Diabetes und Schlaganfall
}

Unter den vaskulären Erkrankungen tritt der Schlaganfall epidemiologisch zunehmend in den Vordergrund. In der bevölkerungsbasierten Studie aus Oxford [1] rangierte er auf „Platz 1 “ der akuten vaskulären Ereignisse knapp vor den kardiovaskulären und deutlich vor den peripher-arteriellen Manifestationen. Vor allem der demographische Wandel sorgt für steigende Inzidenzzahlen nicht nur beim Schlaganfall, sondern auch beim Diabetes. Der Diabetes stellt neben arteriellem Hypertonus und Vorhofflimmern einen der Hauptrisikofaktoren für einen Schlaganfall dar und verdoppelt bis vervierfacht das Risiko. Ein Diabetespatient ist statistisch ebenso schlaganfallgefährdet, wie ein Nicht-Diabetespatient, der bereits einen Schlaganfall erlitten hat, und ein Diabetes führt zu einem etwa 10bis 15-jährigen „zerebrovaskulärenVorausaltern“. Da sich die durch den Diabetes verursachten Schäden am vaskulären „Endorgan Gehirn“ in Studien meist in der Kategorie der „kardiovaskulären“ Diabeteskomplikationen „verstecken“, stellt Prof. Heckmann den unheilvollen ätiologischen Zusammenhang zwischen Diabetes und speziell dem Schlaganfall und die entsprechenden epidemiologischen Risikodaten heraus.

\section{( Der Diabetes stellt einen der Hauptrisikofaktoren für einen Schlaganfall dar}

Die Übersicht von Prof. Sander widmet sich der Primärprävention des Schlaganfalls unter dem Blickwinkel der metabolischen Besonderheiten des Diabetes. Danach erweisen sich „intensive“ und "multimodale“ Interventionen durchaus als hochwirksam, besonders eine konsequente antihypertensive Behandlung. Bei der Sekundärprävention des Schlaganfalls würde man keinen prinzipiellen Unterschied der Wirksamkeit der unterschiedlichen Maßnahmen im Vergleich zur Primärprävention erwarten; dennoch ist hier die Datenlage anders akzentuiert. Die Arbeitsgruppe von Prof. Diener hat dementsprechend die Eckpfeiler einer leitliniengerechten Schlaganfall-Sekundärprävention zusammengestellt und dabei auch die brandaktuellen Daten der RE-LY-Studie integriert [2], die dafür sprechen, dass beim Schlaganfallrisikofaktor Vorhofflimmern erstmals dem „Goldstandard“ der präventiven oralen Antikoagulation mit Phenprocoumon bzw. Warfarin ernste Konkurrenz erwachsen könnte.

Die Übersicht von PD Dr. Ringleb widmet sich der A. carotis als Schaufenster für den makroangiopathischen Gefäßstatus („Intima-Media-Dicke“) beim Diabetespatienten und der damit verbundenen Risikostratifizierung. Effektivität und Evidenzbasiertheit der beiden invasiven Interventionen Stent-Angioplastie (CAS) vs. Karotisendarteriektomie (CEA) werden kritisch analysiert mit dem Ergebnis, dass der vordergründig „minimal-invasive“ Eingriff beim Karotis-Stenting nicht automatisch eine Minimierung des periprozeduralen Risikos bedeutet. Bei der Behandlung symptomatischer Karotisstenosen über 70\% gemäß NASCET-Kriterium bleibt im Moment der therapeutische Goldstandard die CEA.

Ein eigener Beitrag betrachtet differenziert die grundsätzlich negative prognostische Bedeutung einer Hyperglykämie in der Schlaganfall-Akutphase („Post-Stroke-Hyperglykämie“, PSH) und interpretiert die widersprüchlichen Studiendaten zu Nutzen oder Schaden einer strengen glykämischen Kontrolle. Für ein „Laissez-faire“ in der Behandlung der PSH gibt es keinen Anlass! Der Schlüssel zur wirkungsvollen Prognoseverbesserung durch eine strenge Glykämiekontrolle dürfte in der strikten Vermeidung von Hypoglykämien liegen. Kontinuierliche Monitoringsysteme mit bedarfsadaptierter Insulinzufuhr könnten diesen pathophysiologisch richtigen Therapieansatz auf „sicherere Füße" stellen.

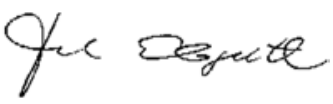

F. Erbguth

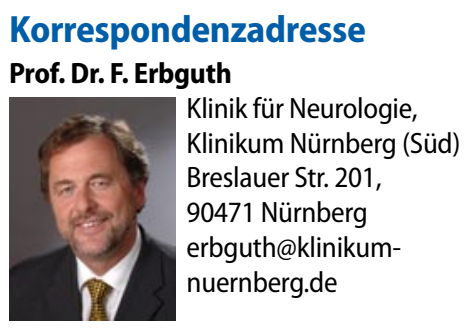

\section{Literatur}

1. Rothwell PM, Coull AJ, Silver LE et al (2005) Population-based study of event-rate, incidence, case fatality, and mortality for all acute vascular events in all arterial territories (Oxford Vascular Study). Lancet 366:1773-1783

2. Connolly SJ, Ezekowitz MD, Yusuf S et al, RE-LY Steering Committee and Investigators (2009) Dabigatran versus warfarin in patients with atrial fibrillation. N Engl J Med 361:1139-1151 
Hier steht eine Anzeige.

算 Springer 


\section{Hier steht eine Anzeige.}

Springer 